\title{
Pseudomelanosis of the stomach
}

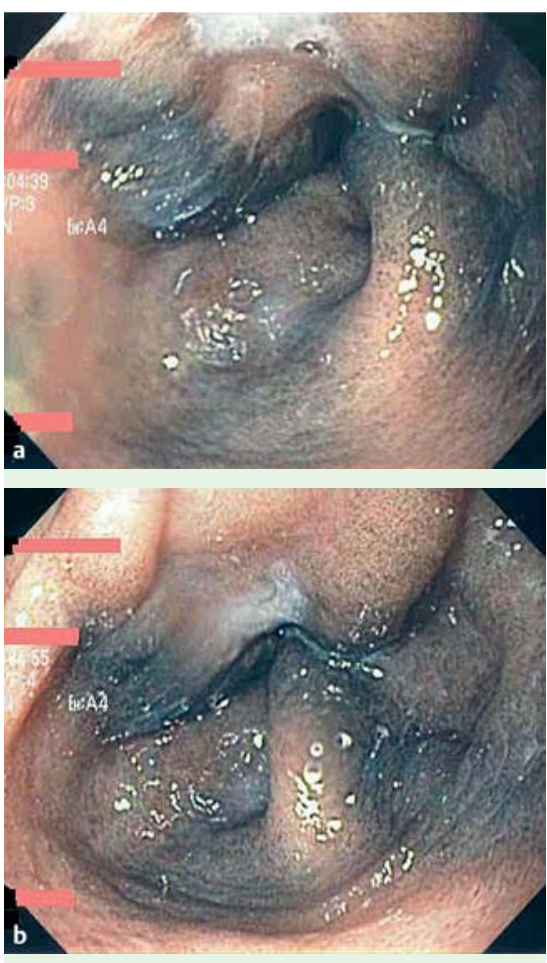

Fig. 1 a, b Endoscopic views showing dark pigmentation of the pyloric antrum.

A 79-year-old woman was seen in consultation for postprandial epigastric pain and early satiety of 2 months' duration, along with weight loss of $4.5 \mathrm{~kg}$ (10 lb). Her history included depression, hypertension, congestive heart failure, and iron deficiency anemia without overt bleeding. Medications included bupropion, escitalopram oxalate, ferrous sulfate, carvedilol, and furosemide. Seven months earlier, an upper and lower endoscopy for the workup of iron deficiency anemia was unremarkable. Upper endoscopy now revealed dark pigmentation of the pyloric antral mucosa (๑ Fig. 1).

The remainder of the gastric mucosa appeared normal. Biopsy specimens revealed brown pigment deposition within macrophages in the lamina propria (๑ Fig. 2).

A positive Prussian blue stain indicated hemosiderin deposition ( $\mathbf{F i g}$. 3).

HMB-45 staining for melanoma and a computed tomography (CT) scan of the abdomen were unremarkable. A gastrointestinal cause for the symptoms could not be found, and severe depression was considered an etiologic factor. Among the patient's risk factors for pseudomelanosis

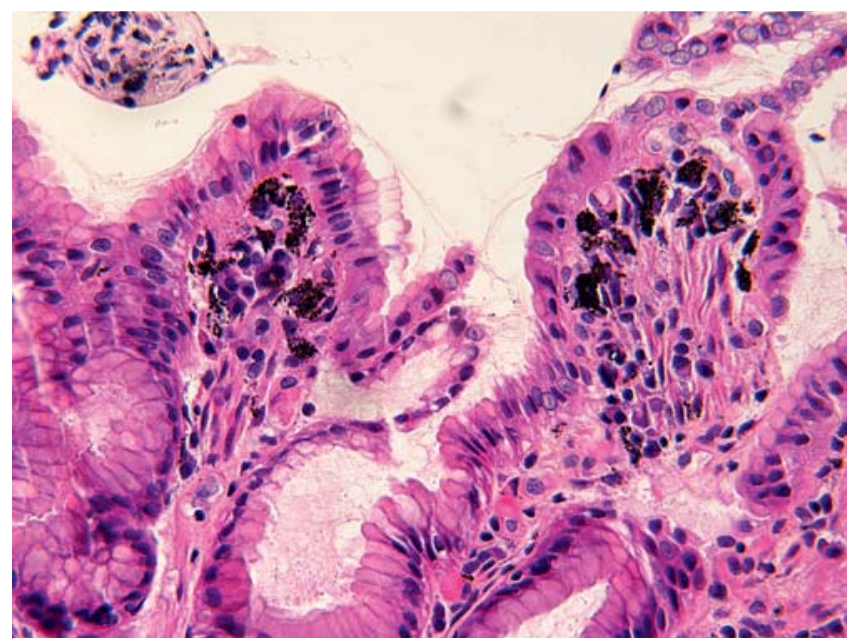

Fig. 2 High magnification view $(\times 400)$ of stained gastric mucosa showing a collection of cells in the lamina propria containing brown pigment.

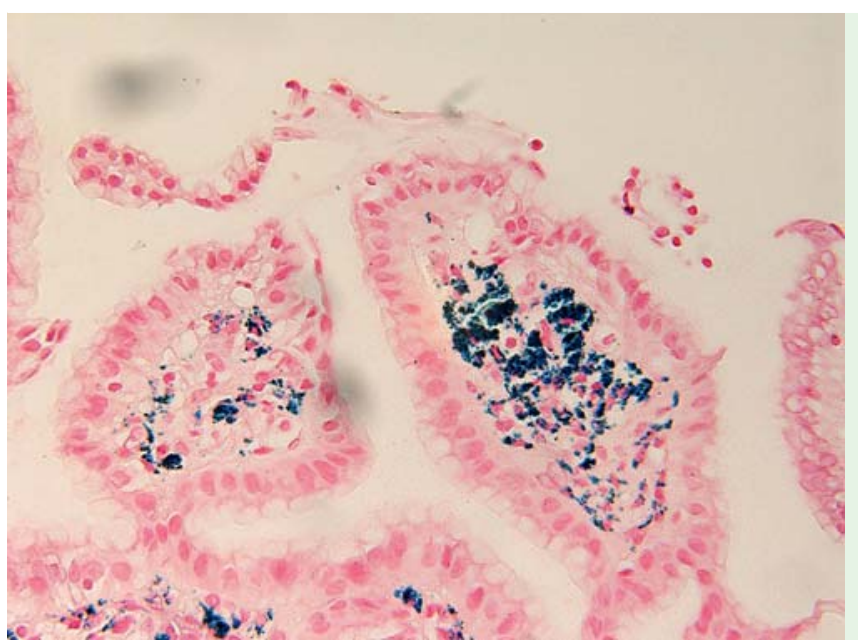

Fig. 3 High magnification view $(\times 400)$ of Prussian-blue-stained gastric mucosa, showing a positive reaction (blue coloration of the pigment).

was the use of ferrous sulfate supplements, which have been implicated in the development of pseudomelanosis.

Gastric pseudomelanosis is extremely rare and the literature is limited to two case reports [1,2]. Iron deposition has been associated with gastrointestinal bleeding, hemochromatosis, chronic renal failure, enteric iron, and several antihypertensive medications [3]. This case report strengthens the association between pseudomelanosis of the stomach and oral iron therapy. Although a rare entity, physicians should be aware of it to facilitate a prompt diagnosis and avoid unnecessary testing.

Endoscopy_UCTN_Code_CCL_1AB_2AD_3AF

\section{R. Kibria, C. J. Barde}

Department of Gastroenterology,

Wright State University School

of Medicine, Dayton, Ohio, USA

\section{References}

1 Weinstock LB, Katzman D, Wang HL. Pseudomelanosis of stomach, duodenum and jejunum. Gastrointest Endosc 2003; 58: 578

2 Antaki F, Irwin BC, Levi E. Rare occurrence of gastric pseudomelanosis. Gastrointest Endosc 2009; 69: 599

3 Moore JD, Baichi M, Toledo R, Sitrin M. Pseudomelanosis of jejunum and ileum. Gastrointest Endosc 2007; 66: 857-859

\section{Bibliography}

DOI $10.1055 / \mathrm{s}-0029-1243871$

Endoscopy 2010; 42: E60

(c) Georg Thieme Verlag KG Stuttgart · New York . ISSN 0013-726X

\section{Corresponding author \\ R. Kibria, MD}

Department of Gastroenterology

Wright State University School of Medicine

4100 W Third Street

Dayton

Ohio 45428

USA

Fax: +937-268-4737

rekibria@gmail.com 